\title{
Optimisation of Heat Exchanger Network Cleaning Schedules Incorporating Uncertainty in Fouling and Cleaning Model Parameters
}

\author{
Riham Al Ismailia ${ }^{\mathrm{a}}$, Min Woo Lee ${ }^{\mathrm{b}}$, D. Ian Wilson ${ }^{\mathrm{a}}$, Vassilios S. Vassiliadis ${ }^{\mathrm{a}, *}$ \\ ${ }^{a}$ Department of Chemical Engineering and Biotechnology, University of Cambridge, West Cambridge Site, \\ Philippa Fawcett Drive, Cambridge CB3 0AS, United Kingdom \\ ${ }^{b}$ Department of Chemical Engineering, Keimyung University, 1095 Dalgubeol-daero, Dalseo-gu, Daegu \\ 42601, South Korea
}

\begin{abstract}
The optimisation of the cleaning schedule in heat exchanger networks (HENs) subject to fouling is presented in which the impact of parametric uncertainty is considered. This work is based on the realisation that these HEN cleaning scheduling problems are multistage mixedinteger optimal control problems (MIOCPs) in which the controls, i.e. cleaning actions, exhibit bang-bang behaviour as they occur linearly in the system equations. A multiple scenario feasible path MIOCP approach is proposed, whereby many scenarios of the HEN multiperiod problem are stacked, sharing the same control actions. This is implemented on a 10 unit and a 25 unit HEN case studies representing crude oil refinery preheat trains (PHTs). Results show that there is a large difference in financial performance for the deterministic case versus the parametric uncertainty problem and hence it is vital that uncertainty be taken into account during the optimisation of schedules for HEN maintenance problems.

Keywords: Optimal control problem; bang-bang control; fouling; parametric uncertainty;
\end{abstract}

\section{Introduction}

Fouling is the deposition and accumulation of undesirable insulating material on the surfaces of heat exchangers. These deposits restrict heat transfer, resulting in loss of efficiency and operability. In studies from the 1980s to the early 1990s, the cost of heat exchanger fouling due to cleaning, fluid treatment, additional hardware and loss of production has been estimated at $0.25 \%$ of the gross domestic product (GDP) of industrialised countries (Müller-Steinhagen et al., 2005). Fouling-related costs in the UK based on the year 1984 were estimated to be in the range of 700-930 million USD (Müller-Steinhagen, 2000). The loss of efficiency in heat exchangers is offset through process turndown or increased utility

\footnotetext{
${ }^{*}$ Corresponding Author

Email address: vsv20@cam.ac.uk (Vassilios S. Vassiliadis)
} 
consumption which results in a surge in greenhouse gas emissions until operational requirements such as temperature and pump-around targets are met. In cases where fouling-related pressure drop changes reduce throughput plant shutdown is often required.

Economic losses are more significant in larger heat exchanger networks which are associated with long continuous operational times, specifically in the PHTs on crude distillation units in oil refineries. Costs linked to crude oil fouling in PHTs worldwide were estimated to be in the order of 4.5 billion USD p.a. according to 1995 figures (Pugh et al., 2002). The addition of antifoulant chemicals, using more robust heat transfer equipment and regular cleaning of fouled units are among the techniques used to alleviate fouling. Cleaning of heat exchangers involves taking units offline, resulting in an increase in operational costs. These cleaning costs can be minimised through optimisation strategies which improve heat transfer of the network over time. Such strategies have been proposed by Ma and Epstein (1981); Sheikh et al. (1996); Smaïli et al. (1999); Georgiadis and Papageorgiou (2000); Lavaja and Bagajewicz (2004b); Ishiyama et al. (2011), among others.

The cleaning scheduling problem is a nonlinear discrete decision-making problem of combinatorial nature where continuous and binary decision variables co-exist, resulting in a mixed-integer nonlinear programming (MINLP) problem. In this problem, time is discretised into periods which are further discretised into cleaning and operating subperiods. Here, a decision must be made to determine which period cleaning should occur and which unit is to be cleaned. This MINLP problem has been solved in a number of ways: (i) Smaïli et al. (2001) used Mathematical Programming (MP) techniques involving discretisation of time into periods, however this approach some times suffers from failure in convergence; (ii) Georgiadis and Papageorgiou (2000) and Lavaja and Bagajewicz (2004b) reformulated the MINLP model into a mixed integer linear programming (MILP) model, but this approach may be computationally expensive and involve the introduction of unsuitable approximations to models; (iii) Smaïli et al. (2002a) used stochastic optimisation frameworks, though this solution method is not guaranteed to be optimal; and (iv) Smaïli et al. (2001); Ishiyama et al. 
(2009)and Gonçalves et al. (2014) used the greedy algorithm, but this scheme is inefficient as it does not consider all the cleaning options over the complete horizon.

In the literature on deterministic optimisation, such problems are classified as linear programming (LP), nonlinear programming (NLP), MILP and MINLP depending on decision variables, objectives and constraints. In reality, perfect forecast of the future is not possible and should be considered uncertain. Stochastic optimisation involves optimal decision making with uncertainties in the data or the model (Diwekar, 2003).

The performance of heat exchangers is directly impacted by uncertainties in data. Babuška and Silva (2014) reported that heat exchangers are typically over-designed by 70-80\%, with 30-50\% of which being attributed to fouling. Fouling is subject to many factors with inherent uncertainty which can be systematic (e.g. the form of the fouling model and whether it should incorporate deposit ageing) and quasi-random (e.g. variation in processed fluid composition over time). Extending lab results to real systems is not straightforward (Smaïli et al., 2002b; Wilson et al., 2017).

In practice, a range of likely fouling rates must be considered when performing scheduling calculations. The areas where parametric uncertainty arise in models for predicting the rate of fouling in crude oil have been highlighted by Wilson et al. (2017):

(i) The type of fouling model that will be observed in a unit cannot be predicted a priori. This is due to the semi-empirical nature of the fouling models as well as the unestablished relationship to crude oil composition and characteristics;

(ii) Fouling models constructed from reconciliation of plant fouling data are commonly used. These are associated with measurement and calculation uncertainties leading to inaccurate fouling rate data;

(iii) In the majority of applications there is variation in the fouling rate over time due to changes in the crude being processed. The relationship between fouling rates and crude composition (e.g. source, blending) is not currently known.

Heat exchanger design is highly reliant on physical properties, e.g. density, specific heat 
capacity, viscosity and thermal conductivity, for estimating heat transfer coefficients and therefore performing overall heat transfer coefficient calculations. Such physical properties are susceptible to a certain degree of uncertainty due to measurement errors, extrapolation and interpolation errors, estimation errors, etc. Furthermore, in the standard methods for heat exchanger analysis such as the number of transfer units (NTU)-effectiveness method, the overall heat transfer coefficient is assumed to be constant and uniform throughout the heat exchanger. In practice this coefficient changes along the length of the heat exchanger and is strongly dependent on a number of factors including the fluid thermo-physical properties and the flow Reynolds number. In a viscous liquid heat exchanger, the heat transfer coefficient can vary by 10 -fold when the flow pattern encompasses laminar, transition and turbulent regions on one side leading to the variation of the overall heat transfer coefficient (Sharqawy and Zubair, 2010). Prasad et al. (2002) highlighted that experimental and/or empirical data for heat transfer coefficients are usually subject to large uncertainties of over $50 \%$ for overall heat transfer coefficients.

These features are evident in figure 1, which shows a time series of the fouling resistance calculated for an industrial shell-and-tube heat exchanger in fouling service. The instantaneous fluctuation arise from the uncertainties in measurement, data, collection and calculation. Pseudo-linear trends are evident between each cleaning event, but the gradient (the fouling rate) differs in each case.

The scheduling of cleaning actions in HENs is highly dependent on fouling model parameters and figure 1 demonstrates how there can be considerable uncertainty in these inputs. Although general scheduling problems with uncertainty have been considered by several authors (Lusa et al., 2008; Galetakis et al., 2012; El Amraoui and Mesghouni, 2014; Hu and Hu, 2016; Kenan et al., 2017; Sel et al., 2017), there has been limited work on HEN cleaning scheduling under uncertainty. A search through the literature found a few publications, such as Lavaja and Bagajewicz (2004a, 2005). Lavaja and Bagajewicz (2004a) extended a reformulated MILP model for the planning of HEN cleaning in chemical plants to include uncertainty 


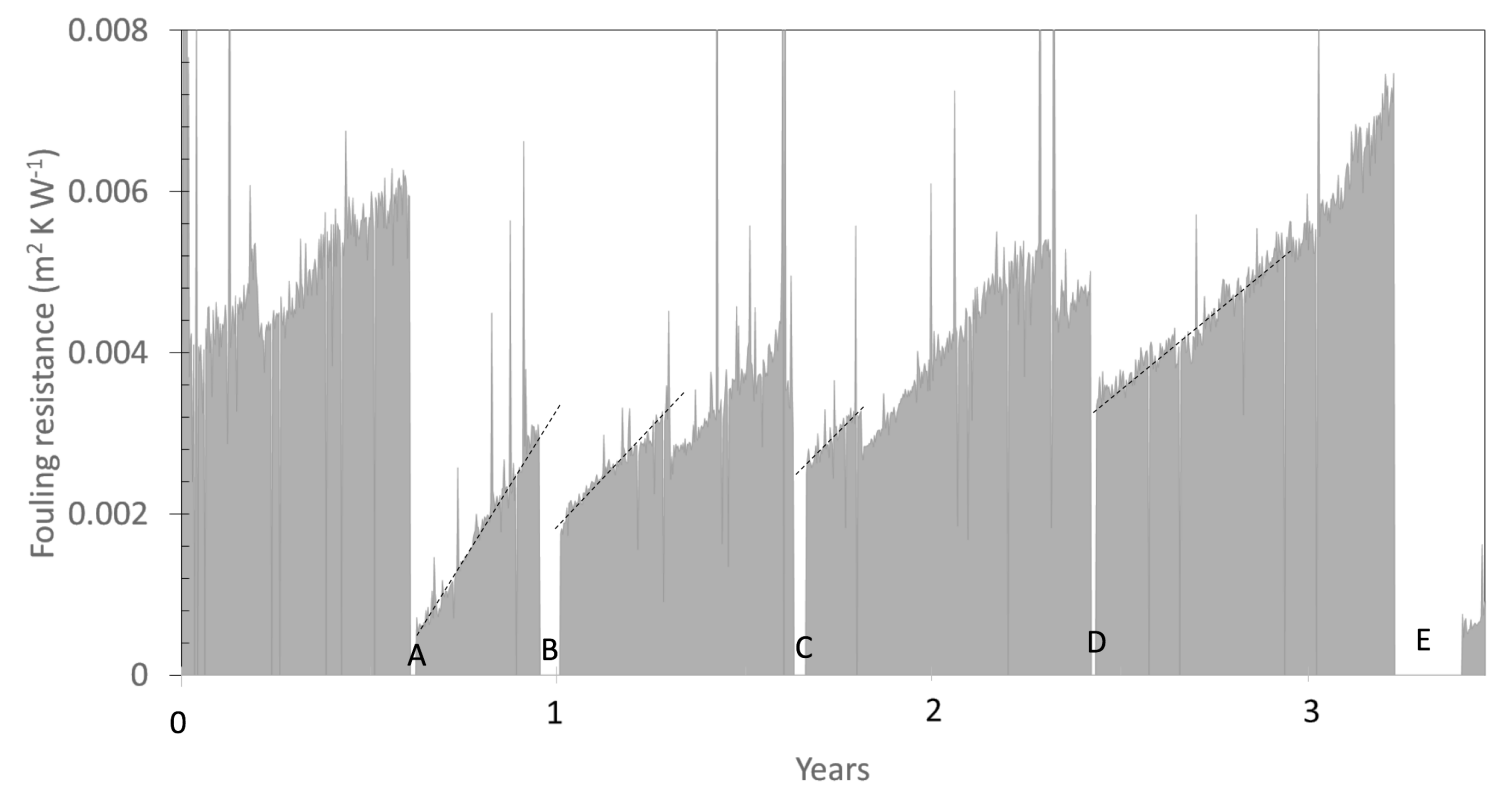

Figure 1: Evolution of fouling resistance in a refinery heat exchanger over a 40 month period. Letters indicate when the unit was cleaned. Rigorous cleaning was performed at A and E: less intensive cleaning at B, C and D. Dashed lines show simple linear fits to data following cleaning. Reproduced from Ishiyama et al. (2017).

in the data. Although the fouling coefficients, cleaning costs, plant turnaround horizons and the processing of different crudes at different times are uncertain parameters, they only considered uncertainty in energy prices. Lavaja and Bagajewicz (2004a) used the standard two-stage stochastic programming model, which was developed by Beale (1955); Dantzig (1955). They constructed the scenarios through sampling energy prices. The sampling was done assuming normal distributions, with increasing standard deviation for similar months of the year as the time horizon increases. They compared their stochastic solutions to deterministic and heuristic solutions and discussed financial risk management options. Lavaja and Bagajewicz (2005) studied the effects of uncertainty in parameters, including the future fuel price used in the furnace, the change of feedstock and the fouling rate of the crude processed, on the cleaning schedule. They used a reformulated MILP model coupled with a multi-scenario based approach and showed how the optimal cleaning schedule can vary when different parameters are considered uncertain simultaneously. Furthermore, their results showed how the model helped determine the best cleaning schedule to apply when risk is involved. 
In our recent work on an optimal control approach to HEN cleaning scheduling we showed that HEN cleaning scheduling problems are in actuality MIOCPs which exhibit a nearly bangbang solution (Al Ismaili et al., 2018). In this work a stochastic optimisation version of these models is developed for the first time and applied to crude oil PHTs in oil refineries. The paper is organised as follows: Section 2 describes the formulation as a multi-period optimal control problem (OCP) and presents the general HEN cleaning scheduling problem. The approach for HEN cleaning scheduling with parametric uncertainty is presented in section 3. Implementation and solutions to two case studies are presented in sections 4 and 5.: (i) a network consisting of 10 heat exchangers and (ii) a larger network of 25 units. A commercial optimisation software is used.

\section{Solution procedure: HEN cleaning scheduling without uncertainty}

Equations (1a) to (1d) show the general formulation for an OCP in which the performance index is minimised subject to control, $u(t)$ and differential algebraic equations (DAEs). These equations describe an index-1 differential algebraic equation (DAE) system where an initial condition, $x_{0}$ and a fixed final time, $t_{\mathrm{F}}$, are given. The problem considered involves differential and algebraic state variables $x(t)$ and $y(t)$, respectively, as well as binary control variables, $u(t)$, hence it is termed a MIOCP.

$$
\min _{u(\cdot)} O b j=\phi\left[x\left(t_{\mathrm{F}}\right)\right]+\int_{0}^{t_{\mathrm{F}}} L[x(t), y(t), u(t), t] d t
$$

subject to

$$
\begin{gathered}
\dot{x}(t)=f[x(t), y(t), u(t), t], \quad x\left(t_{0}\right)=x_{0}, \\
g(x(t), y(t), u(t), t)=0,
\end{gathered}
$$




$$
u(t) \in \mathcal{U}, \quad \mathcal{U} \in\{0,1\} \quad \forall t \in\left[0, t_{\mathrm{F}}\right]
$$

For the OCP solution procedure, time is discretised into a number of periods, $p=$ $1,2, \ldots, N P$, in which $N P$ is the total number of periods. Period lengths have not been specified. Here, there are discontinuities present within the control profiles at a finite number of time points, $t_{p}$, where these are the points in time where period $p$ ends and is termed a junction. A general form of junction conditions between stages, i.e. periods $p$ and $p+1$ is given in equation (2) (Vassiliadis, 1993).

$$
\begin{gathered}
J_{p}\left(\dot{x}_{p+1}\left(t_{p}^{+}\right), x_{p+1}\left(t_{p}^{+}\right), y_{p+1}\left(t_{p}^{+}\right), u_{p+1}\left(t_{p}^{+}\right), \dot{x}_{p}\left(t_{p}^{-}\right), x_{p}\left(t_{p}^{-}\right), y_{p}\left(t_{p}^{-}\right), u_{p}\left(t_{p}^{-}\right), t_{p}\right)=0 \\
\forall p=1,2, \ldots, N P-1
\end{gathered}
$$

The basic formulation of a multi-period OCP over time periods, $p=1,2, \ldots, N P, t \in$ $\left[t_{p-1}, t_{p}\right]$ with $t_{N P}=t_{\mathrm{F}}$ is displayed in equations $(3 \mathrm{a})$ to $(3 \mathrm{~g})$.

$$
\min _{u(\cdot)} \operatorname{Obj}=\sum_{p=1}^{N P}\left[\phi^{(p)}\left(x^{(p)}\left(t_{p}\right), y^{(p)}\left(t_{p}\right), u^{(p)}, t^{(p)}\right)\right]+\int_{t_{p-1}}^{t_{p}} L^{(p)}\left[x^{(p)}(t), y^{(p)}(t), u^{(p)}, t\right] d t
$$

subject to

$$
\begin{gathered}
\dot{x}^{(p)}(t)=f^{(p)}\left(x^{(p)}(t), y^{(p)}(t), u^{(p)}, t\right) \\
0=g^{(p)}\left(x^{(p)}(t), y^{(p)}(t), u^{(p)}, t\right) \\
t_{p-1} \leq t \leq t_{p}, \quad p=1,2, \ldots, N P
\end{gathered}
$$




$$
\begin{gathered}
x^{(1)}\left(t_{0}\right)=I^{(1)}\left(u^{(1)}\right) \\
x^{(p)}\left(t_{p-1}\right)=I^{(p)}\left(x^{(p-1)}\left(t_{p-1}\right), y^{(p-1)}\left(t_{p-1}\right), u^{(p)}\right) \quad \forall p=2,3, \ldots, N P \\
u(t) \in \mathcal{U}, \quad \mathcal{U} \in\{0,1\}
\end{gathered}
$$

For the HEN cleaning scheduling problem the controls $u^{(p)}(t)$ are considered to be piecewise constant so as to reflect the on/off nature of a unit being cleaned or not. The stage switching times $t_{p}$ are fixed in this initial derivation and period lengths are equal. The collective vector of controls over all stages is described in equation (4). Junction conditions allow differential state variables to be reset based on the value of the control variable, as shown in equation (5).

$$
\begin{gathered}
\boldsymbol{u}=\left(u^{(1)}, u^{(2)}, \ldots, u^{(N P)}\right)^{T} \\
x^{(p)}\left(t_{p-1}\right)=u^{(p)}(t) \cdot x^{(p-1)}\left(t_{p-1}\right) \quad \forall p=2, \ldots N P
\end{gathered}
$$

The control in the relaxed multistage MIOCP for cleaning scheduling is linearly related to the process variables. When this occurs, the optimal control for the relaxed MIOCP will exhibit bang-bang behaviour (with potential singular arcs). Bang-bang solutions occur when the optimal control action is at either bound of the feasible region (Bryson and Ho, 1975). Controls that are not bang-bang, where the control lies between the bounds, are called singular arcs. Pure bang-bang controls have been reported in a variety of applications (Bellman et al., 1956; Blakemore and Aris, 1962; Mohler, 1973; Belghith et al., 1986; Alt et al., 2016; Yan and Zhu, 2017).

Sager (2009) reviewed approaches for optimal control of nonlinear dynamical systems with 
binary controls (on/off). For bang-bang optimal control behaviour, the general OCP is reformulated such that the binary controls are presented linearly in the system dynamics. It may be required to use heuristics, e.g. rounding up, a sum up rounding strategy, or algorithms such as Branch and Bound when singular arcs appear.

The scheduling cleaning problem does not require this reformulation as the controls involved are already presented linearly in the system. Discretisation of the system equations is unnecessary, as formulating this problem as an OCP aids the solution of the relaxed NLP problem through the feasible path (i.e. sequential) approach, which otherwise leads to a very large scale optimisation problem with a strongly nonlinear system of equality constraints.

The HEN scheduling optimisation formulation is presented as follows:

The overall heat transfer coefficient, $U$, is related to the fouling resistance, $R_{\mathrm{f}}$ by:

$$
\frac{1}{U}=\frac{1}{U_{\mathrm{c}}}+R_{\mathrm{f}}
$$

where subscript c denotes the clean condition.

A number of different fouling behaviours are encountered in practice. Two common ones are considered here, namely linear and asymptotic forms:

$$
\begin{gathered}
\dot{R}_{\mathrm{f}}=a \\
R_{\mathrm{f}}=R_{\mathrm{f}}^{\infty}\left(1-\exp \left(-t^{\prime} / \tau\right)\right)
\end{gathered}
$$

where $a$ is the linear fouling rate, $R_{f}^{\infty}$ is the asymptotic fouling resistance, $\tau$ is the decay constant and $t^{\prime}$ is the operating time elapsed since the last cleaning action. The values of the parameters can vary between exchangers in a PHT due to differences in bulk and wall temperature and design.

Using the the log-mean temperature difference method, the heat duty of a shell and tube heat exchanger is given by:

$$
Q=U A G \triangle T_{\mathrm{lm}}
$$


Here, $A$ is the area, $\triangle T_{l m}$ is the logarithmic mean temperature difference and $G$ is the configuration geometry factor. In this work, $G$ is set to unity, corresponding to pure coor counter-current flow. The full optimisation model development follows the derivation presented in Al Ismaili et al. (2018), and is shown in Appendix A.

\section{Solution procedure: HEN cleaning scheduling with parametric uncertainty}

A similar formulation to the one presented in the previous section, where the problem is formulated as an OCP, is used for the HEN maintenance scheduling problem with parametric uncertainty along with a few modifications:

(i) Multiple scenarios, $s$, where $s \in S$, are created by generating random values normally distributed about the mean of each of the uncertain parameters $\left(\bar{a}, \bar{R}_{f}^{\infty}, \bar{\tau}, \bar{U}_{c}\right.$ and $\left.\bar{C}_{E}\right)$, i.e. a totally random sampling of space and not a systematic one. This is achieved using the normrnd function in MATLAB $^{\circledR}$.

(ii) The objective function is modified from equation (A.4) to equation (10), where this is now a summation of the mean operating cost from all scenarios and the cleaning cost.

$$
O b j=\left(\sum_{s=1}^{S} \int_{0}^{t_{\mathrm{F}}} \frac{C_{\mathrm{E}, s} Q_{\mathrm{F}, s}(t)}{\eta_{f}} d t\right) / S+\sum_{p=1}^{N P} \sum_{n=1}^{N E} C_{c l}\left(1-y_{n, p}\right)
$$

(iii) The same control action is applied to all scenarios, as given in equation (10).

A multiple scenario based approach is proposed due to its simplicity and direct implementation to the MIOCP approach. Here, a simulation is used to evaluate a batch of scenarios based on the level of uncertainty in each of the parameters in question. An analysis of the effect of the number of scenarios produced in this scheduling cleaning problem is conducted. The impact of uncertainty in the fouling rate parameters, which includes the linear fouling constant, $a$, asymptotic fouling resistance, $R_{f}^{\infty}$ and decay constant, $\tau$, on the HEN cleaning scheduling problem is assessed. The sensitivity of the overall cost due to fouling to the overall heat transfer coefficient in the clean condition, $U_{\mathrm{c}}$ and fuel cost, $C_{\mathrm{E}}$, are also analysed. This 
analysis is performed by considering uncertainty in one parameter at a time whilst the other parameters are fixed. For the parametric uncertainty problem, uncertainty is considered in all of the aforementioned parameters simultaneously.

This multiple scenario approach gives a larger DAE system by stacking many realisations of the HEN multiperiod problem whilst applying the same control action among all scenarios. In the simulation phase, the DAE integrator is overloaded while the optimisation problem remains at the same size as a single scenario presented to the optimiser. Here, the number of decision variables is the same among the multiple scenarios which are solved using the feasible path approach. Hence, the optimiser is presented with effectively an unconstrained problem, whether a single-scenario case is used or a multiple-scenario case. The only constraints that may be present in these formulations are the ones that are associated with the binary variables which are not dependent on the number of scenarios. This highlights the potency of the proposed feasible path methodology, in that it can be used effectively to tackle the maintenance scheduling problem with uncertainty. This strategy is used for the sake of keeping both the optimisation problem size under control, as well as contributing to reducing computational time to a practical level. Computational time can be further reduced owing to this approach being highly parallelisable.

The implementation is performed in MATLAB ${ }^{\circledR}$ R2016b with its Optimisation Toolbox ${ }^{\text {TM }}$ and Parallel Computing Toolbox ${ }^{\text {TM }}$ (The MathWorks Inc., 2016). The MATLAB ${ }^{\circledR}$ code works as a standard multi-period optimal control problem solver using the feasible path approach by linking together the Ordinary Differential Equation (ODE) solver ode15s with the optimiser fmincon. The default settings for ode15s are used, with absolute tolerance of $10^{-6}$ and relative tolerance of $10^{-3}$. The optimiser fmincon is used with the Sequential Quadratic Programming (SQP) algorithm option whilst keeping the remaining settings at their default values: constraint, optimality and step tolerances of $10^{-6}$ using a forward finite difference scheme for the calculation of gradients. Gradient evaluations conducted via finite differences are costly and require repeated simulations of the dynamic process model. The 
Parallel Computing Toolbox ${ }^{\mathrm{TM}}$ in MATLAB ${ }^{\circledR}$ is used for parallelisation of the gradient evaluations. All case studies are performed on a 4GHz Intel Core i7, 16 GB RAM iMac running on macOS Sierra. Single optimisations are performed for each scenario, i.e. from a single starting point. Here, all the control variables' initial points are set to 1 .

\section{Case studies}

Computational experiments for the scheduling of cleaning actions for HENs with parametric uncertainty in the form of in crude oil PHTs undergoing fouling are considered here.

We present a network of 10 units appearing in the work of Lavaja and Bagajewicz (2004b) (Case I) and a more complex network of 25 units presented by Smaïli et al. (2002a) (Case II). These are shown in figures 2 and 3. Stream data for each model are presented in tables 1 and 3. Case I employs 18 periods $(N P=18)$ while Case II employs 36 periods $(N P=36)$. The selection and operational constraints imposed on each of these cases are summarised in tables 2 and 4, respectively. Both linear and asymptotic fouling models are considered in Case I whilst only linear fouling is modelled in Case II.

The scheduling problem was reformulated into a MILP problem by Lavaja and Bagajewicz (2004b) whereas Smaïli et al. (2002a) solved the MINLP problem directly using two methods: a Backtracking Threshold Accepting (BTA) stochastic search algorithm and the Outer Approximation (OA) method.

There is no mention of the furnace fuel cost in the work of Lavaja and Bagajewicz, so a $\bar{C}_{\mathrm{E}}$ of $£ 2.93 / \mathrm{MM}$ Btu is used in Case I which is based on the value reported by Smaïli et al. (2002b). The work of Smaïli et al. (2002b) is the source of data for Lavaja and Bagajewicz's models where they compared the solutions from their MILP approach with those obtained by Smaïli et al. (2002b) using an OA/ER algorithm. For Case II, a $\bar{C}_{\mathrm{E}}$ of $£ 0.34 / \mathrm{kW}$ day is used. For Case I, although Lavaja and Bagajewicz stated that they accounted for the decay in the heat transfer coefficient in each sub-period, expressed by $\eta_{c}$, there is no mention of the value of this parameter in their work. Hence, we considered the value of parameter $\eta_{c}$ to be 
1 in our model. This decay parameter is also fixed at the value of 1 in Case II along with the furnace efficiency $\eta_{f}$. Smaïli et al. (2002a) did not consider these parameters in their model. The mean cleaning cost incurred for cleaning operations, $\bar{C}_{c l}$, is $£ 4000$ per cleaning action in Case I and $£ 5000$ for Case II. For the latter case, the duration of the cleaning and operating sub-periods are equal with $\triangle t^{\mathrm{CL}}=\Delta t^{\mathrm{OP}}=15$ days.

For Case I, sensitivity analysis of the overall cost to the following parameters is performed: linear and asymptotic fouling parameters $\left(a, R_{f}^{\infty}\right.$ and $\left.\tau\right)$; the overall heat transfer coefficient in the clean condition $\left(U_{\mathrm{c}}\right)$; and the furnace fuel cost $\left(C_{\mathrm{E}}\right)$. Additionally, for this case, the impact of the number of scenarios $(S)$ on the schedule is considered. Relative standard deviation (RSD) values of 5, 10, 15 and 20\% around the mean of each of the parameters examined are considered while the range $10 \leq S \leq 50$ is considered for the study of the impact of the number of scenarios. For the parametric uncertainty problem, a single realisation is performed in each of the case studies. 


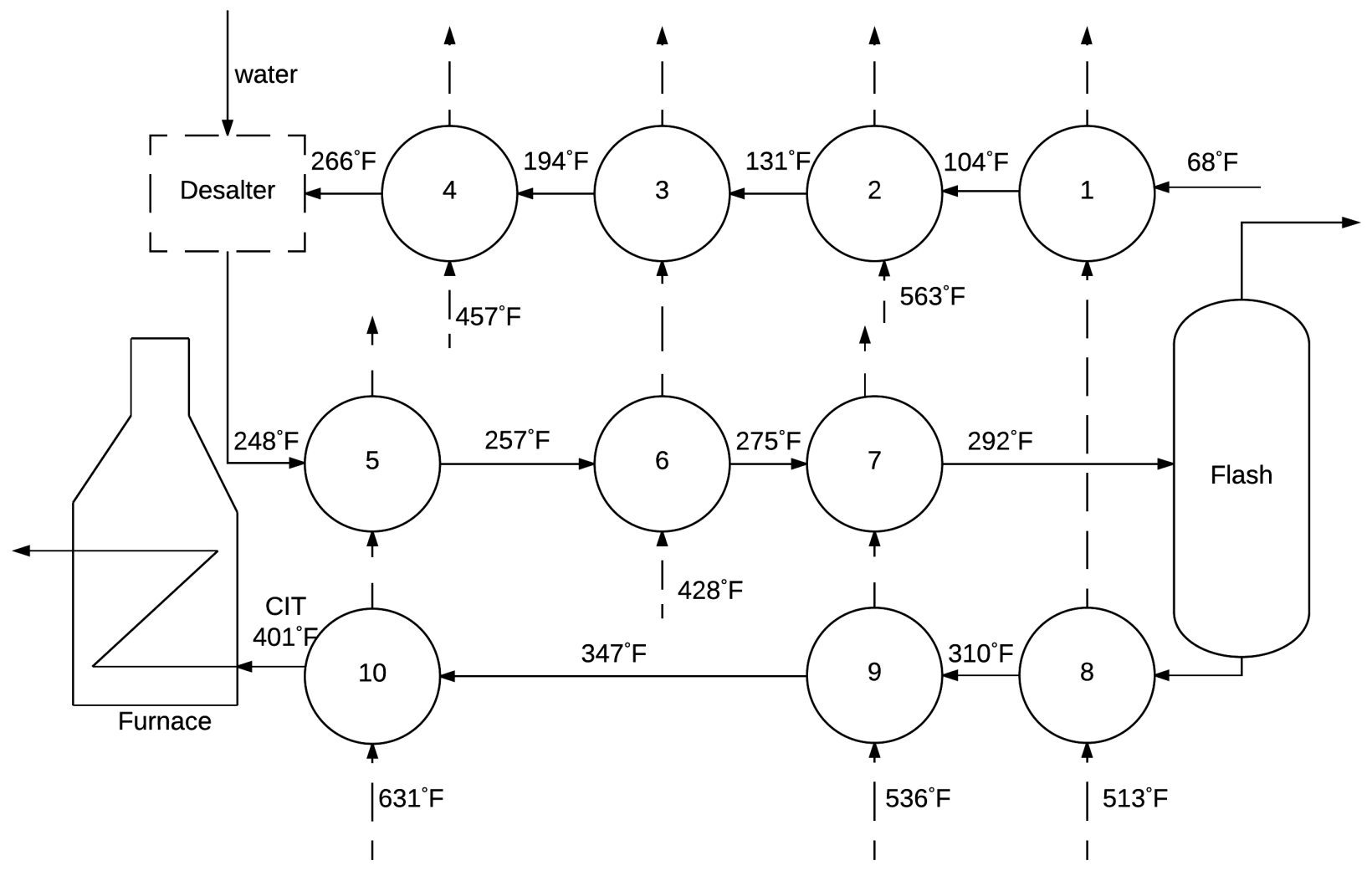

Figure 2: Flow diagram for Case I, 10 unit HEN. Temperature values are given for initial, clean condition. Adapted from Lavaja and Bagajewicz, 2004b. 


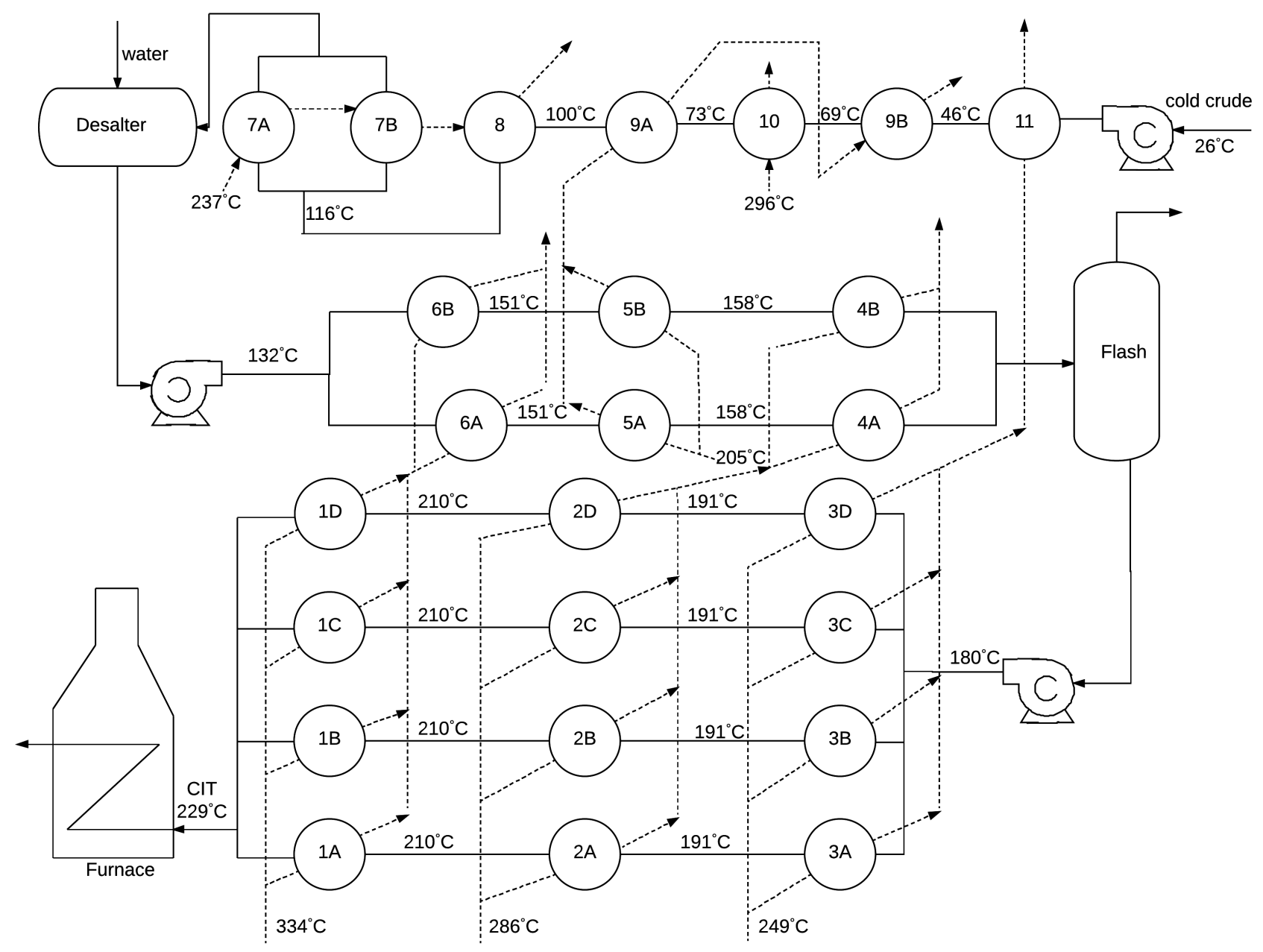

Figure 3: Flow diagram for Case II, 25 unit HEN case. Solid lines, cold (crude) streams; dashed lines, hot streams; CIT, crude inlet temperature to furnace. Temperature values are given for initial, clean condition. Adapted from Smaïli et al., 2002a.

\section{Computational results}

\subsection{Case I}

For Case I, the RSD for each uncertain parameter is fixed at $10 \%$ when the impact of number of samples on the schedule is assessed. For the case with linear fouling, the deterministic cost due to fouling i.e. the optimal cost without uncertainty, is $£ 260 \mathrm{k}$. Results for the impact of the number of samples with linear fouling are shown numerically in table 5. 


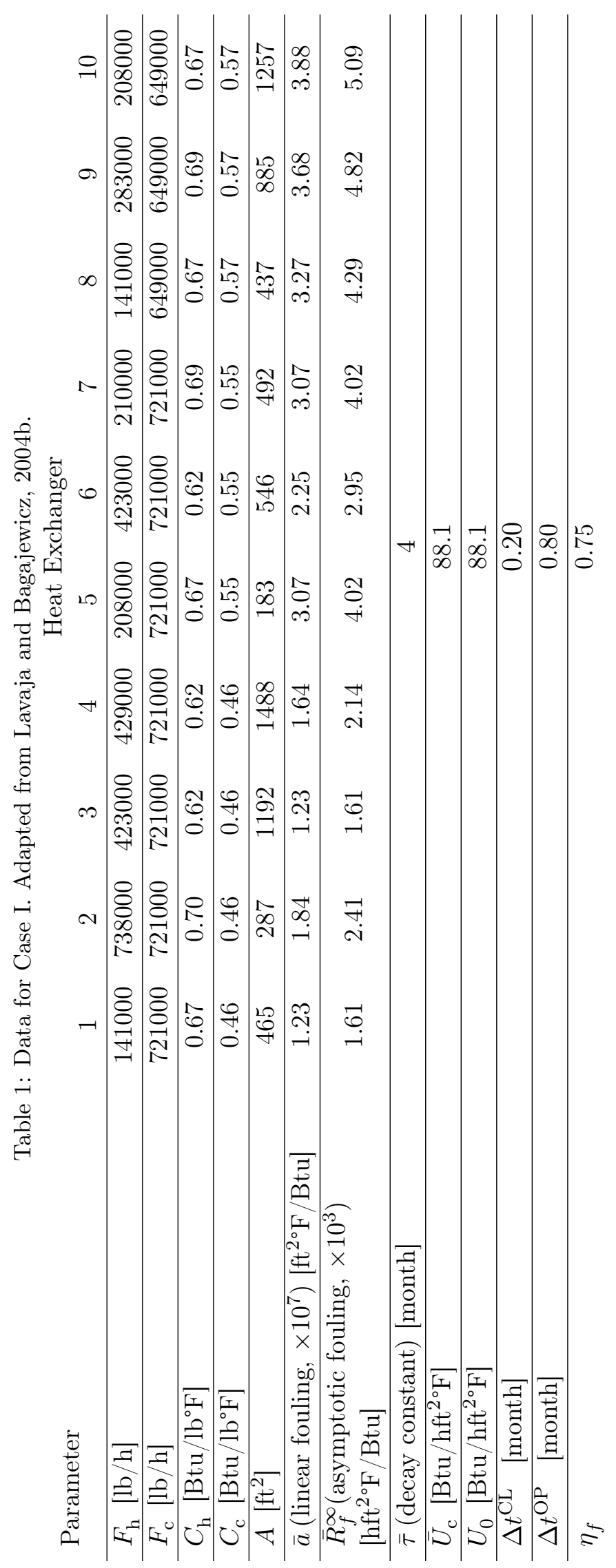


Table 2: Operational constraints for Case I. Adapted from Lavaja and Bagajewicz, 2004b.

only one unit of exchangers $1-4$ can be cleaned in each period $\quad y_{1 p}+y_{2 p}+y_{3 p}+y_{4 p} \geq 3 \forall p$ only one unit of exchangers $5-7$ can be cleaned in each period $\quad y_{5 p}+y_{6 p}+y_{7 p} \geq 2 \forall p$ temperature drop across desalter $\quad T_{\mathrm{c}, 5 p}^{\mathrm{in}}=T_{\mathrm{c}, 4 p}^{\text {out }}-18 \forall p$

Table 3: Data for Case II. Adapted from Smaïli et al., 2002a.

\begin{tabular}{|c|c|c|c|c|c|c|c|}
\hline$H E X$ & $\begin{array}{l}F_{\mathrm{h}} \\
\left(\mathrm{kg} \mathrm{s}^{-1}\right)\end{array}$ & $\begin{array}{l}F_{\mathrm{c}} \\
\left(\mathrm{kg} \mathrm{s}^{-1}\right)\end{array}$ & $\begin{array}{l}C_{\mathrm{h}} \\
\left(\mathrm{kJ} \mathrm{kg}^{-1} \mathrm{~K}^{-1}\right)\end{array}$ & $\begin{array}{l}C_{\mathrm{c}} \\
\left(\mathrm{kJ} \mathrm{kg}^{-1} \mathrm{~K}^{-1}\right)\end{array}$ & 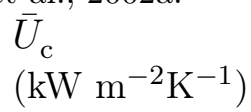 & $\begin{array}{l}A \\
\left(\mathrm{~m}^{2}\right)\end{array}$ & $\begin{array}{l}\bar{a} \times 10^{11} \\
\left(\mathrm{~m}^{2} \mathrm{KJ}^{-1}\right)\end{array}$ \\
\hline $1 \mathrm{~A}$ & 8.7 & 23 & 2.8 & 2.4 & 0.5 & 21.3 & 1.9 \\
\hline $2 \mathrm{~A}$ & 11.4 & 23 & 2.9 & 2.4 & 0.5 & 29.7 & 1.8 \\
\hline $3 \mathrm{~A}$ & 4.8 & 23 & 2.8 & 2.4 & 0.5 & 31.4 & 1.6 \\
\hline $1 \mathrm{~B}$ & 8.7 & 23 & 2.8 & 2.4 & 0.5 & 21.3 & 1.9 \\
\hline $2 \mathrm{~B}$ & 11.4 & 23 & 2.9 & 2.4 & 0.5 & 29.7 & 1.8 \\
\hline $3 \mathrm{~B}$ & 4.8 & 23 & 2.8 & 2.4 & 0.5 & 31.4 & 1.6 \\
\hline $1 C$ & 8.7 & 23 & 2.8 & 2.4 & 0.5 & 21.3 & 1.9 \\
\hline $2 \mathrm{C}$ & 11.4 & 23 & 2.9 & 2.4 & 0.5 & 29.7 & 1.8 \\
\hline $3 \mathrm{C}$ & 4.8 & 23 & 2.8 & 2.4 & 0.5 & 31.4 & 1.6 \\
\hline $1 \mathrm{D}$ & 8.7 & 23 & 2.8 & 2.4 & 0.5 & 21.3 & 1.9 \\
\hline $2 \mathrm{D}$ & 11.4 & 23 & 2.9 & 2.4 & 0.5 & 29.7 & 1.8 \\
\hline $3 \mathrm{D}$ & 4.8 & 23 & 2.8 & 2.4 & 0.5 & 31.4 & 1.6 \\
\hline $4 \mathrm{~A}$ & 23 & 47.4 & 2.8 & 2.3 & 0.5 & 26.7 & 1.5 \\
\hline $5 \mathrm{~A}$ & 28 & 47.4 & 2.6 & 2.3 & 0.5 & 35.4 & 1.1 \\
\hline $6 \mathrm{~A}$ & 17.4 & 47.4 & 2.9 & 2.3 & 0.5 & 79.1 & 1.5 \\
\hline $4 \mathrm{~B}$ & 23 & 47.4 & 2.8 & 2.3 & 0.5 & 29.2 & 1.6 \\
\hline $5 \mathrm{~B}$ & 28 & 47.4 & 2.6 & 2.3 & 0.5 & 35.4 & 1.1 \\
\hline $6 \mathrm{~B}$ & 17.4 & 47.4 & 2.9 & 2.3 & 0.5 & 79.1 & 1.5 \\
\hline $7 \mathrm{~A}$ & 25 & 47.4 & 2.6 & 1.92 & 0.5 & 60.8 & 0.8 \\
\hline $7 \mathrm{~B}$ & 25 & 47.4 & 2.6 & 1.92 & 0.5 & 80.3 & 0.8 \\
\hline 8 & 49.6 & 95 & 2.6 & 1.92 & 0.5 & 129 & 0.8 \\
\hline $9 \mathrm{~A}$ & 55.8 & 95 & 2.6 & 1.92 & 0.5 & 110 & 0.9 \\
\hline $9 \mathrm{~B}$ & 55.8 & 95 & 2.6 & 1.92 & 0.5 & 96.6 & 0.9 \\
\hline 10 & 3.3 & 95 & 2.9 & 1.92 & 0.5 & 8.5 & 0.6 \\
\hline 11 & 19.1 & 95 & 2.8 & 1.92 & 0.5 & 56.6 & 0.6 \\
\hline
\end{tabular}




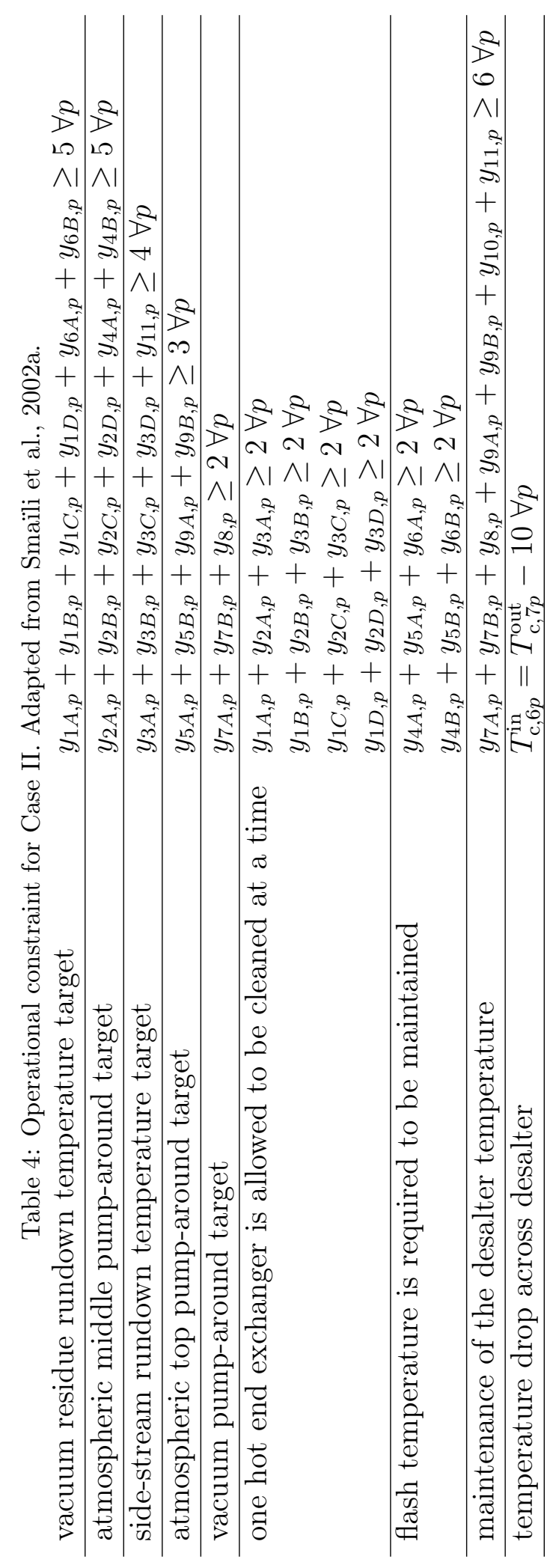


There is no direct correlation between mean overall cost due to fouling and number of samples. Although the mean cost increases by $£ 62 \mathrm{k}$ when the number of samples are increased from 10 to 40, this value decreases by $£ 8 \mathrm{k}$ from 40 to 50 samples (see table 5 ). This is because of the way in which the sample sets are generated. The set of samples are independent from each other, i.e. they are not produced by the addition of 10 more samples to the previous set repeatedly. Sample sets are created in the same manner for the asymptotic fouling case. The costs due to fouling for each scenario are broadly dispersed about their mean value. Here, the RSD ranges from $31.1 \%$ up to $53.7 \%$, with the largest deviation corresponding to the least number of samples. In addition, the difference between maximum and minimum cost for each scenario is large, ranging from $£ 321 \mathrm{k}$ to $£ 500 \mathrm{k}$, as shown in table 5 . The total number of cleanings for the deterministic case is 11, while this ranges from 9 to 11 cleanings with a mode of 10 cleanings when the number of samples are varied.

The sensitivity analysis for each of the fouling, overall heat transfer coefficient and fuel cost parameters is performed with a fixed number of samples, of 30. Results concerning the sensitivity of the overall cost due to fouling to the linear fouling rate, $a$, show that the sensitivity of the cost to this parameter is limited. Table 6 shows that the mean cost varies by only up to $£ 4 \mathrm{k}$ when the deviation of the linear fouling rate about its mean is increased from $5 \%$ to $20 \%$.

Moreover, the results are narrowly dispersed about the mean cost, where the variation in the RSD value lies within the range of $1.1 \%$ to $4 \%$. The largest difference between the maximum and minimum costs is $£ 45.6 \mathrm{k}$ for the $20 \%$ deviation case. The variation in the number of cleanings between each case is small, increasing from 10 cleanings for the $5 \%$ and $10 \%$ deviation scenarios to 11 for the $15 \%$ and $20 \%$ scenarios (see table 6 ).

Table 7 shows that the cost due to fouling is sensitive to $C_{\mathrm{E}}$; the RSD increases from $4.7 \%$ to $15.5 \%$ as the deviation of the fuel cost parameter is risen from $5 \%$ to $20 \%$. Furthermore, the difference between the maximum and minimum value of the overall cost due to fouling increases by more than a factor of 3 , from $£ 47.4 \mathrm{k}$ to $£ 159 \mathrm{k}$. The overall costs due to fouling 
are more scattered in this parameter than the linear fouling rate. In this scenario, the mean cost due to fouling increases by $£ 12 \mathrm{k}$, from $£ 261 \mathrm{k}$ to $£ 273 \mathrm{k}$, when the deviation of the fuel cost parameter is increased from $5 \%$ to $20 \%$.

As expected, the maximum cost increases when the deviation of $C_{\mathrm{E}}$ is increased. However, although the minimum cost decreases, from $£ 157 \mathrm{k}$ to $£ 67 \mathrm{k}$, when the deviation of $C_{\mathrm{E}}$ is increased from $5 \%$ to $10 \%$, this value increases to $£ 109 \mathrm{k}$ when the deviation is increased by $10 \%$ further. Generally, the minimum cost should decrease with increase in deviation for well sampled scenarios. This is also observed in the results for the sensitivity of the cost due to fouling to $U_{\mathrm{c}}$ (see table 8 ) and in the asymptotic case (see tables 12 to 15 ). In contrast to the impact of $a$ on the schedule, the number of cleanings here does not change with the level of deviation in $C_{\mathrm{E}}$ and remains constant at 10 cleanings.

Results for the sensitivity of the cost due to fouling to the overall heat transfer coefficient in the clean condition, shown in table 8, reveal that the cost associated with the HEN cleaning scheduling problem is highly sensitive to this parameter. The RSD increases from $17.2 \%$ to $60.7 \%$ when the deviation of this parameter is increased from $5 \%$ to $20 \%$. Moreover for each scenario, there is a large difference between the maximum and minimum cost, e.g. over 7 times and over 16 times in the $10 \%$ and $20 \%$ deviation scenarios, respectively. Generally, the number of cleaning actions required here remain constant at 10 and only increases by 1 in the $20 \%$ deviation scenario.

For the case with parametric uncertainty, we show one realisation where each of $a, U_{\mathrm{c}}$ and $C_{\mathrm{E}}$ parameters are considered uncertain simultaneously. Here, we set the deviation of each of these parameters to $10 \%$ and the number of samples to 30 . The results in table 9 show the P90, P50 and P10 cost values. The P90 is the cost level with $10 \%$ probability of exceeding and $90 \%$ probability to under run. This value is $£ 397 \mathrm{k}$, as shown in table 9 , and is considered a conservative estimate of the cost due to fouling. P10 is the cost value with $90 \%$ probability of exceeding and $10 \%$ probability to under run; this more optimistic value is $£ 168 \mathrm{k}$. The P50 cost, also known as the $50^{\text {th }}$ percentile, is the median and is $£ 276 \mathrm{k}$. In 
Table 5: Effect of number of samples for Case I (linear fouling).

No. of samples

\begin{tabular}{llllll} 
& 10 & 20 & 30 & 40 & 50 \\
\hline Mean cost [k£] & 234 & 274 & 276 & 296 & 288 \\
\hline RSD [\%] & 53.7 & 34.3 & 31.1 & 34.6 & 36.0 \\
\hline Minimum cost [k£] & 40.8 & 94.0 & 87.1 & 98.9 & 43.7 \\
\hline Maximum cost [k£] & 523 & 519 & 408 & 546 & 544 \\
\hline No. of cleanings & 9 & 10 & 10 & 11 & 10 \\
\hline
\end{tabular}

this case, this value is equivalent to the mean cost due to fouling resulting in a normal curve, i.e. symmetrical distribution of a cost against probability curve with no skew, as shown in figure 4. The associated full width at half maximum (FWHM) and standard deviation (SD) is $£ 202 \mathrm{k}$ and $£ 86 \mathrm{k}$, respectively. The weighted average cost is the sum of all outcomes times the respective probabilities, and given that we have a normal curve, this value is very similar to that of the P50 with only £1k difference, as shown in table 9. Overall, the anticipated cost associated with this realisation is $£ 277 \mathrm{k} \pm 31.1 \%$ in comparison to a cost of $£ 260 \mathrm{k}$ for the deterministic case.

Table 10 compares the cleaning schedules for the linear fouling case with and without parametric uncertainty. All exchangers except unit 2 are cleaned the same number of times in each scenario. In the parametric uncertainty case, unit 2 is not cleaned at all, while it is cleaned once in the deterministic case. There is a limited number of common cleaning actions with only 1 for unit 10 during the $12^{\text {th }}$ month. Moreoever, the parametric uncertainty cleaning actions sometimes take place earlier than the deterministic ones and other times later. However, these cleanings are always within 2 periods of each other.

In terms of resource usage, convergence for the deterministic problem of the linear fouling case required $446 \mathrm{CPU}$ s (7.4 CPU min) over 13 SQP major iterations and 2,269 function evaluations, i.e. simulations (this number includes simulations required for finite difference calculations). The corresponding problem with parametric uncertainty required $\approx 36$ times more resource usage totalling 16,160 CPU s (4.5 CPU hr) with 16 SQP major iterations and 2,921 function evaluations. 
Table 6: Sensitivity of linear fouling constant for Case I.

Deviation of linear fouling constant [\%] $\begin{array}{llll}5 & 10 & 15 & 20\end{array}$

\begin{tabular}{lllll}
\hline Mean cost $[\mathrm{k} £]$ & 262 & 266 & 265 & 266 \\
\hline RSD [\%] & 1.1 & 2.2 & 3.2 & 4.0 \\
\hline Minimum cost $[\mathrm{k} £]$ & 257 & 254 & 247 & 247 \\
\hline Maximum cost in $[\mathrm{k} £]$ & 267 & 275 & 279 & 293 \\
\hline No. of cleanings & 10 & 10 & 11 & 11 \\
\hline
\end{tabular}

Table 7: Sensitivity of fuel cost parameter for Case I (linear fouling). Deviation of fuel cost parameter [\%]

\begin{tabular}{lllll} 
& 5 & 10 & 15 & 20 \\
\hline Mean cost $[\mathrm{k} £]$ & 261 & 266 & 265 & 273 \\
\hline RSD [\%] & 4.7 & 8.7 & 13.5 & 15.5 \\
\hline Minimum cost $[\mathrm{k} £]$ & 235 & 215 & 180 & 196 \\
\hline Maximum cost $[\mathrm{k} £]$ & 283 & 311 & 338 & 355 \\
\hline No. of cleanings & 10 & 10 & 10 & 10 \\
\hline
\end{tabular}

Table 8: Sensitivity of overall heat transfer coefficient parameter for Case I (linear fouling).

Deviation of overall heat transfer coefficient parameter [\%]

\begin{tabular}{lllll} 
& 5 & 10 & 15 & 20 \\
\hline Mean cost $[\mathrm{k} £]$ & 252 & 269 & 299 & 303 \\
\hline RSD [\%] & 17.2 & 38.2 & 43.0 & 60.7 \\
\hline Minimum cost $[\mathrm{k} £]$ & 157 & 67.4 & 109 & 50.2 \\
\hline Maximum cost in $[\mathrm{k} £]$ & 338 & 483 & 620 & 836 \\
\hline No. of cleanings & 10 & 10 & 10 & 11 \\
\hline
\end{tabular}

Table 9: Cleaning scheduling with parametric uncertainty for Case I (linear fouling).

\begin{tabular}{ll} 
Mean cost [k£] & 276 \\
\hline RSD [\%] & 31.1 \\
\hline FWHM [k£] & 202 \\
\hline SD [k£] & 86 \\
\hline Minimum cost [k£] & 87.1 \\
\hline Maximum cost [k£] & 458 \\
\hline P90 [k£] & 397 \\
\hline P50 [k£] & 276 \\
\hline P10 [k£] & 168 \\
\hline Weighted average cost [k£] & 277 \\
\hline
\end{tabular}




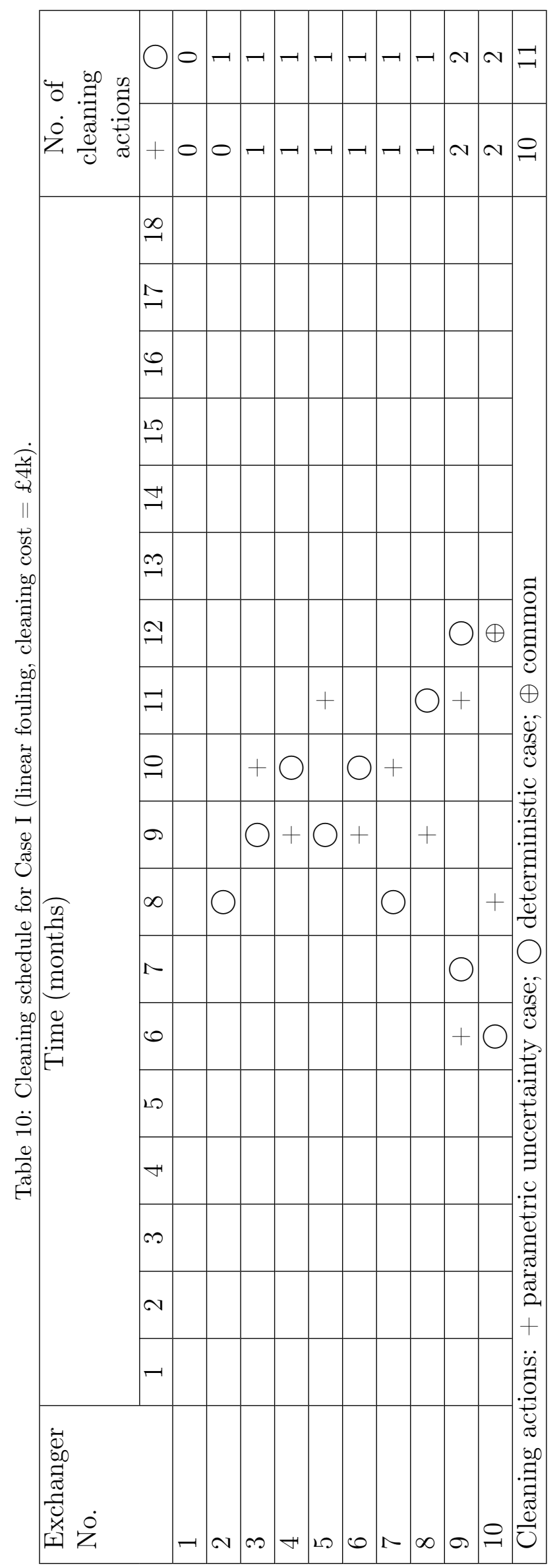




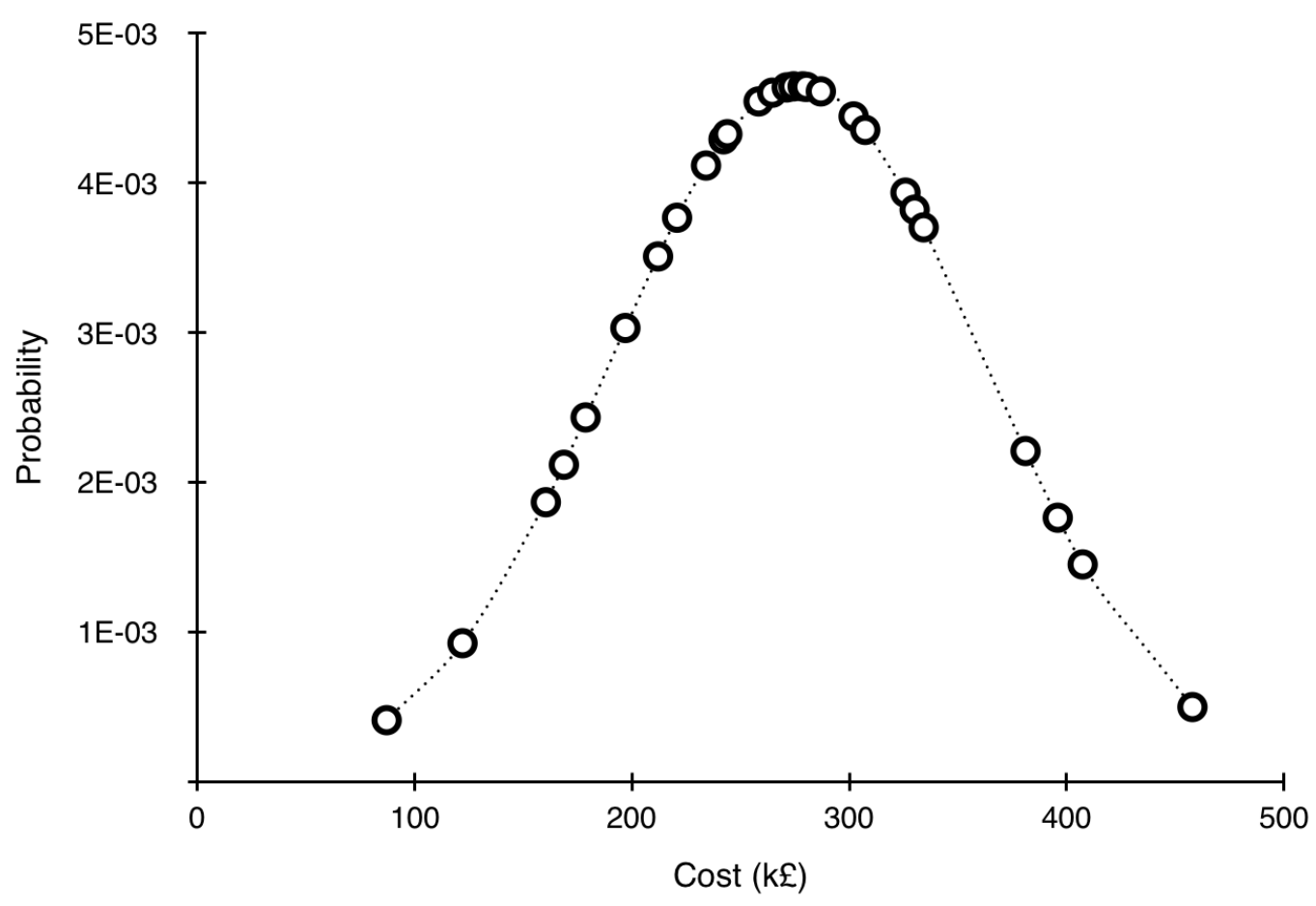

Figure 4: Cost versus probability curve for Case I (linear fouling).

For the asymptotic fouling behaviour scenario a similar procedure is followed, where the deviation of uncertain parameters is fixed at $10 \%$ when the effect of the number of samples is assessed and the number of samples is fixed at 30 when the sensitivity of these parameters is analysed.

Results for the effect of the number of samples on the cost due to asymptotic fouling displayed in table 11 show that the mean cost varies from $£ 476 \mathrm{k}$ to $£ 552 \mathrm{k}$ in comparison to $£ 507 \mathrm{k}$ for the deterministic case. As with the linear fouling case, due to the sample sets being independent from each other, there is no direct relation between the mean cost due to fouling and the number of samples considered. In this case, with every increase of 10 samples there is an increase in the mean cost followed by a decrease in this value, e.g. the mean cost increases by $£ 48 \mathrm{k}$ when the sample size is increased from 10 to 20 , but decreases by $£ 17 \mathrm{k}$ when the sample size is increased further to 30 .

The overall costs due to fouling are scattered about their mean value (see table 11) and 
consequently the RSD varies from $18.2 \%$ to up to $21.8 \%$. In comparison to the linear fouling case, these costs are less dispersed (21.8\% maximum in this case vs. $53.7 \%$ maximum in the linear fouling case), with the largest RSD corresponding to the largest number of samples. The total number of cleaning for the deterministic case and parametric uncertainty case are similar (4 vs. a mode of 3 cleanings).

Numerical results concerning the sensitivity of the cost of fouling to $R_{\mathrm{f}}^{\infty}$ and $\tau$ are shown in tables 12 and 13, respectively. There are a couple of similarities in the results concerning the sensitivity analysis of these asymptotic fouling parameters: (i) The sensitivity of the cost to the asymptotic fouling parameters is limited, such that the mean cost only varies by $£ 13 \mathrm{k}$ and $£ 17 \mathrm{k}$ across a deviation of 5 to $20 \%$ for $R_{\mathrm{f}}^{\infty}$ and $\tau$, respectively (shown in tables 12 and 13) and (ii) there is a tight spread of results about the mean cost, where the RSD reaches a peak of $4.9 \%$ and $7 \%$ when the deviation of $R_{\mathrm{f}}^{\infty}$ and $\tau$ are increased to $20 \%$, respectively.

However, in contrast to the linear fouling constant, there is a larger variation in the number of cleanings required for the asymptotic fouling resistance, where this ranges from 3 up to 6 cleaning actions. This is not the case for the decay constant parameter which shows a limited variation (3 to 4 cleaning actions).

Results for the effect of the fuel cost parameter show that the overall cost is more sensitive to this parameter than the asymptotic fouling parameters. This is shown in table 14, where the RSD varies up to $17.7 \%$ for a deviation of $20 \%$ of $C_{\mathrm{E}}$. In addition, there is an increase in the difference between the maximum and minimum cost of more than 4 times from $£ 78.4 \mathrm{k}$ to $£ 334 \mathrm{k}$ over the range of 5 to $20 \%$ deviation in the fuel cost parameter. Therefore, there is more of a spread in the cost than that associated with the asymptotic fouling parameters. Unlike the linear fouling case, a decrease in the mean cost is observed when the deviation of the fuel cost parameter is increased from $5 \%$ to $20 \%$. The number of cleaning actions required is almost constant and only varies from 3 to 4 in this case.

Results for the sensitivity of the cost to $U_{\mathrm{c}}$ are shown in table 15 . Similarly to the linear fouling case, the HEN scheduling problem's cost is highly sensitive to $U_{c}$, in which the RSD 
is $36.4 \%$ when the deviation of this parameter is increased to $20 \%$ (see table 15). Moreover, the difference between the maximum and minimum cost is over 4 times in the $20 \%$ deviation scenario. The number of cleaning actions required is almost constant (3 to 4).

Furthermore, similarly to the linear fouling case, for the asymptotic fouling problem with parametric uncertainty, we show one realisation where each of $R_{\mathrm{f}}^{\infty}, \tau, U_{\mathrm{c}}$ and $C_{\mathrm{E}}$ are considered uncertain simultaneously. Again, we set the deviation of each of these parameters to $10 \%$ and the number of samples to 30 . The P90, P50 and P10 values are $£ 666 \mathrm{k}, £ 502 \mathrm{k}$ and $£ 401 \mathrm{k}$, respectively, as shown in table 16 . In this case the mean cost is $£ 5 \mathrm{k}$ higher than the P50 value at $£ 507 \mathrm{k}$, resulting in a slightly positively skewed cost against probability curve, as shown in figure 5. As this curve is only slightly skewed, i.e. has an almost symmetrical distribution, the weighted average cost is equal to the P50 value. Hence, the expected cost associated with this realisation is $£ 502 \mathrm{k} \pm 20.6 \%$ in comparison to a cost of $£ 507 \mathrm{k}$ for the deterministic case.

The comparison of the cleaning schedules with and without parametric uncertainty in table 17 shows that only exchangers 9 and 10 are cleaned over the horizon of 18 months. Exchanger 9 is cleaned one less time in the parametric uncertainty case than the deterministic one, giving a total of 3 cleaning actions in the former versus 4 cleaning actions in the latter. There are 2 common cleaning actions taking place just before the midpoint of the horizon.

In terms of resource usage, convergence for the deterministic problem with asymptotic fouling required $461 \mathrm{CPU}$ s (7.7 CPU min) over $11 \mathrm{SQP}$ major iterations and 2,055 function evaluations. Convergence for the parametric uncertainty case required 8,722 CPU s (2.4 CPU hr) with $7 \mathrm{SQP}$ major iterations and 1,370 function evaluations.

\subsection{Case II}

For parametric uncertainty problem of Case II, one realisation is performed where $a$, $U_{\mathrm{c}}$ and $C_{\mathrm{E}}$ are considered uncertain simultaneously. Again, we set the deviation of each of these parameters to $10 \%$ and the number of samples to 30 . The P90, P50 and P10 values are $£ 1140 \mathrm{k}$, $925 \mathrm{k}$ and $£ 804 \mathrm{k}$, respectively, as shown in table (18). The mean cost is $£ 29 \mathrm{k}$ 
Table 11: Effect of number of samples for Case I (asymptotic fouling).

No. of samples

\begin{tabular}{llllll} 
& 10 & 20 & 30 & 40 & 50 \\
\hline Mean cost $[\mathrm{k} £]$ & 476 & 524 & 507 & 552 & 534 \\
\hline RSD [\%] & 18.2 & 16.9 & 20.6 & 17.2 & 21.8 \\
\hline Minimum cost $[\mathrm{k} £]$ & 316 & 352 & 310 & 360 & 327 \\
\hline Maximum cost $[\mathrm{k} £]$ & 635 & 718 & 732 & 795 & 769 \\
\hline No. of cleanings & 4 & 3 & 3 & 3 & 4 \\
\hline
\end{tabular}

Table 12: Sensitivity of asymptotic fouling resistance for Case I.

Deviation of asymptotic fouling resistance [\%]

\begin{tabular}{lllll} 
& 5 & 10 & 15 & 20 \\
\hline Mean cost $[\mathrm{k} £]$ & 508 & 499 & 511 & 512 \\
\hline RSD [\%] & 1.2 & 2.5 & 4.3 & 4.9 \\
\hline Minimum cost $[\mathrm{k} £]$ & 493 & 475 & 454 & 457 \\
\hline Maximum cost $[\mathrm{k} £]$ & 519 & 528 & 543 & 568 \\
\hline No. of cleanings & 3 & 6 & 4 & 3 \\
\hline
\end{tabular}

Table 13: Sensitivity of decay constant for Case I (asymptotic fouling).

Deviation of decay constant [\%]

\begin{tabular}{lllll} 
& 5 & 10 & 15 & 20 \\
\hline Mean cost $[\mathrm{k} £]$ & 508 & 518 & 501 & 508 \\
\hline RSD [\%] & 1.5 & 3.0 & 5.4 & 7.0 \\
\hline Minimum cost $[\mathrm{k} £]$ & 493 & 490 & 424 & 428 \\
\hline Maximum cost $[\mathrm{k} £]$ & 522 & 546 & 552 & 581 \\
\hline No. of cleanings & 4 & 3 & 4 & 3 \\
\hline
\end{tabular}

Table 14: Sensitivity of fuel cost parameter for Case I (asymptotic fouling).

Deviation of fuel cost parameter [\%]

\begin{tabular}{lllll} 
& 5 & 10 & 15 & 20 \\
\hline Mean cost [k£] & 504 & 503 & 498 & 499 \\
\hline RSD [\%] & 4.3 & 9.7 & 13.9 & 17.7 \\
\hline Minimum cost [k£] & 466 & 404 & 307 & 302 \\
\hline Maximum cost [k£] & 545 & 590 & 641 & 620 \\
\hline No. of cleanings & 4 & 4 & 3 & 4 \\
\hline
\end{tabular}


Table 15: Sensitivity of overall heat transfer coefficient parameter for Case I (asymptotic fouling).

Deviation of overall heat transfer coefficient parameter [\%]

\begin{tabular}{lllll} 
& 5 & 10 & 15 & 20 \\
\hline Mean cost [k£] & 523 & 530 & 587 & 515 \\
\hline RSD [\%] & 6.4 & 18.6 & 17.5 & 36.4 \\
\hline Minimum cost $[\mathrm{k} £]$ & 462 & 347 & 411 & 218 \\
\hline Maximum cost $[\mathrm{k}]$ & 590 & 763 & 806 & 895 \\
\hline No. of cleanings & 4 & 4 & 3 & 4 \\
\hline
\end{tabular}

Table 16: Cleaning scheduling with parametric uncertainty for Case I (asymptotic fouling).

\begin{tabular}{ll} 
Mean cost $[\mathrm{k} £]$ & 507 \\
\hline RSD [\%] & 20.6 \\
\hline Minimum cost $[\mathrm{k} £]$ & 310 \\
\hline Maximum cost in $[\mathrm{k} £]$ & 732 \\
\hline P90 [k£] & 666 \\
\hline P50 [k£] & 502 \\
\hline P10 [k£] & 401 \\
\hline Weighted average cost [k£] & 502 \\
\hline
\end{tabular}

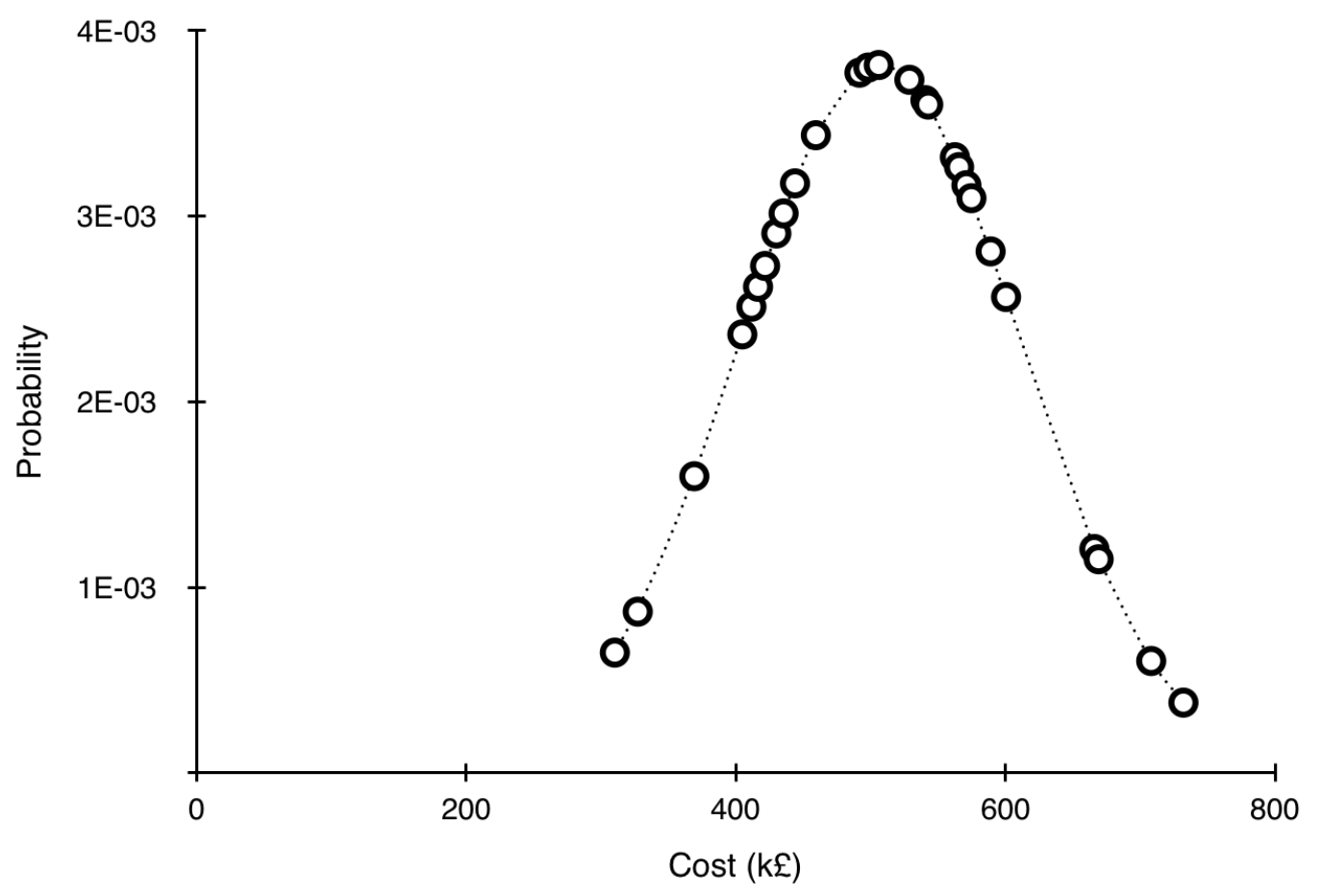

Figure 5: Cost versus probability curve for Case I. 


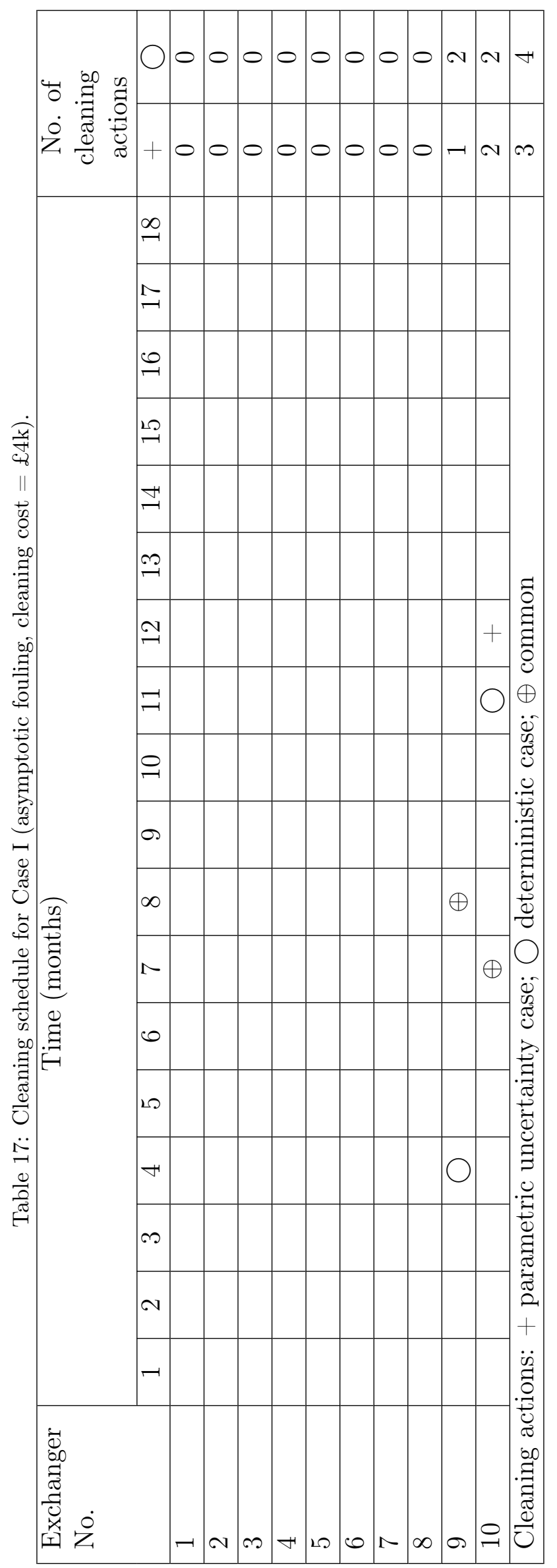


Table 18: Cleaning scheduling with parametric uncertainty for Case II.

\begin{tabular}{ll} 
Mean cost $[\mathrm{k}\rfloor]$ & 954 \\
\hline RSD [\%] & 16.3 \\
\hline Minimum cost $[\mathrm{k} £]$ & 654 \\
\hline Maximum cost in $[\mathrm{k} £]$ & 1305 \\
\hline P90 [k£] & 1140 \\
\hline P50 [k£] & 925 \\
\hline P10 [k£] & 804 \\
\hline Weighted average cost $[\mathrm{k} £]$ & 935 \\
\hline
\end{tabular}

higher than the $\mathrm{P} 50$ value at $£ 954 \mathrm{k}$ resulting in the cost $v s$. probability curve being slightly positively skewed, as shown in figure (6). Here, the weighted average cost is £10k more than the P50 value. Hence, the expected cost associated with this realisation is $£ 935 \mathrm{k} \pm 16.3 \%$ in comparison to a cost of $£ 896 \mathrm{k}$ for the deterministic case.

The cleaning schedules with and without parametric uncertainty displayed in table (19) differ. No pattern is observed here and cleaning actions occur earlier for some units in the parametric uncertainty case in comparison to the deterministic one and later for other units. There are only 6 common cleaning actions out of a total of 39 and 37 cleaning actions for the parametric uncertainty and deterministic cases, respectively.

The resource usage is $29,173 \mathrm{CPU} \mathrm{s}(\approx 8.1 \mathrm{CPU} \mathrm{hr})$ and 1.26 million $\mathrm{CPU} \mathrm{s}(\approx 14.6$ CPU day) for the deterministic and parametric uncertainty cases, respectively. For the former case, convergence required 26 SQP major iterations and 23,696 function evaluations. For the latter case, convergence required 38 SQP major iterations and 34,208 function evaluations. The high time CPU time is attributed to the use of a finite difference scheme for the calculation of gradients which is inefficient. Future work will include the reduction of the CPU time to a more practical value. This will be achieved through the use of sensitivity equations for gradient calculations. 


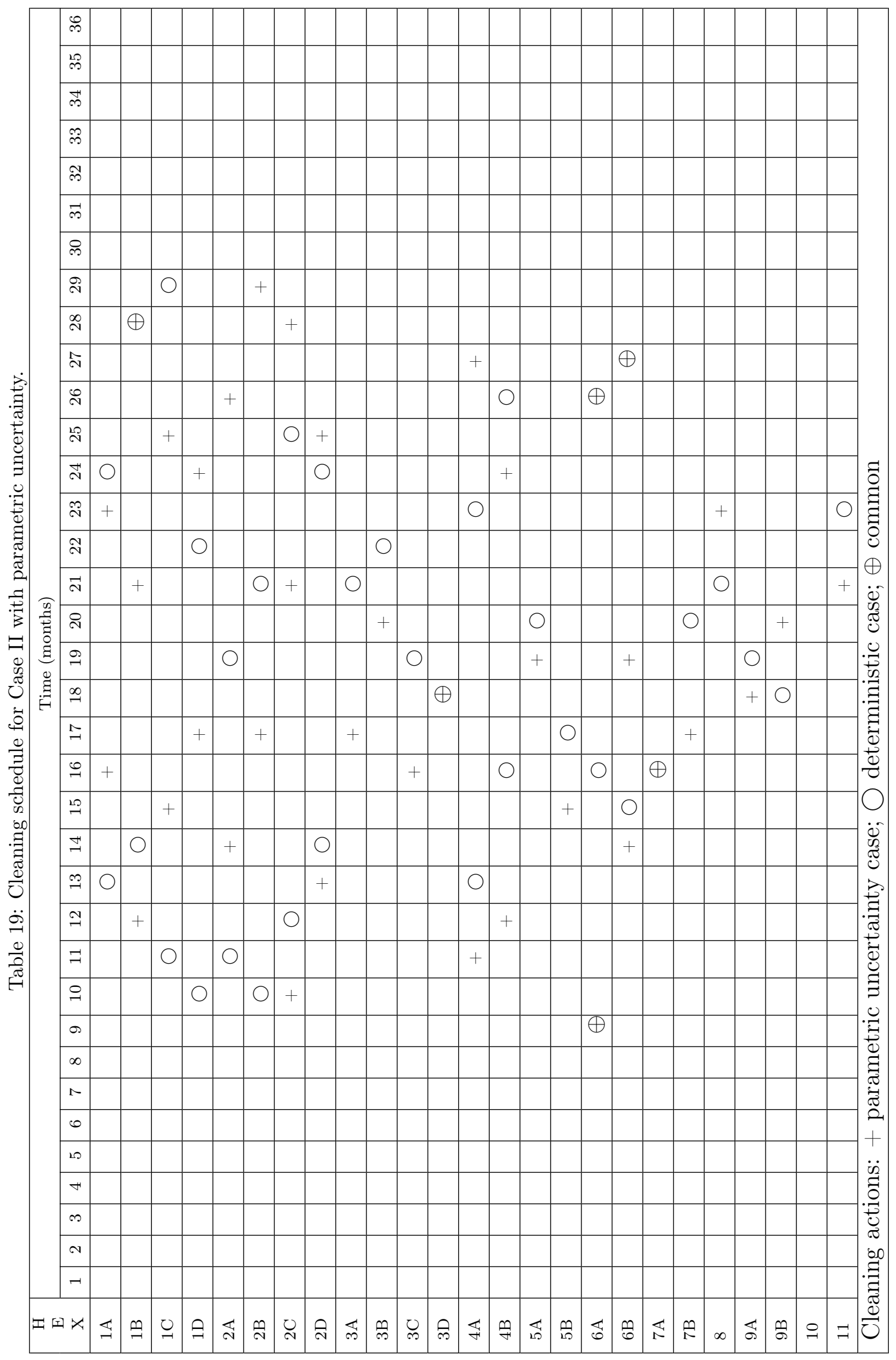




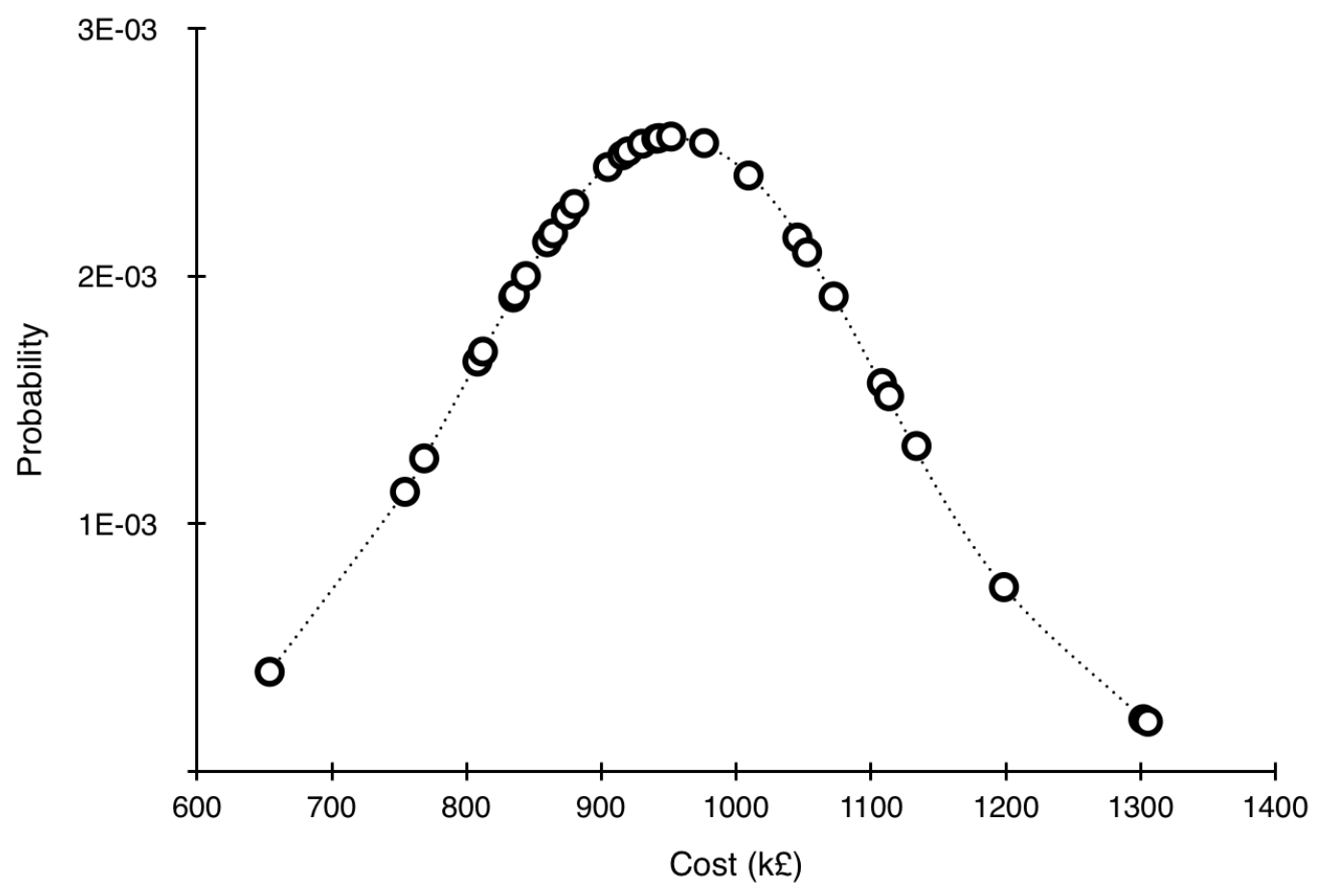

Figure 6: Cost versus probability curve for Case II.

\section{Discussion}

The above case studies have demonstrated how finding that HEN cleaning scheduling problems exhibit bang-bang control characteristics allows the uncertainty associated with fouling behaviour and other input parameters to be incorporated in scheduling calculations. This allows the impact of uncertainty in the fouling behaviour(s) to be quantified alongside the sensitivity of the results to the costing parameters that appear in the objective function. The former can be considered as a 'natural' variation, while the latter can vary simply as a result of budget and market considerations. The latter are also likely to vary between installations owing to differences in network configuration, availability of fuel and other factors.

The methodology presented here therefore allows the significance of each source of uncertainty to be established and the sensitivity of these factors to be compared. For instance, if the source of uncertainty in fouling model parameterisation is dominated by the absence of reliable data, the results can be used to justify the cost of installing new instrumenta- 
tion. Likewise, if the schedule is strongly dependent on the costing parameter, management decisions can be made on the basis of forecasts over the anticipated operating period.

Furthermore, it is important to highlight that the methodology presented in this work is based on an offline scheduling approach. Adaptive scheduling can be used for the HEN cleaning scheduling problem, where estimates for the fouling and cost data can be refined in 'real' time, i.e. online, as one goes along. This will ultimately reduce the impact of uncertainty in these parameters on the overall cost due to fouling.

\section{Conclusions}

In conclusion, for the first time a multiple scenario feasible path MIOCP approach is developed and applied to the scheduling of cleaning actions in HENs which undergo fouling. The parametric uncertainty model runs automatically with ease without any failures. Results show that some parameters, specifically the cost of fuel and the clean overall heat transfer coefficient, have a strong influence on the overall cost due to fouling in HEN maintenance scheduling problems. Consequently, comparison of results with and without parametric uncertainty shows that there is a large difference in economics for the deterministic case versus the parametric uncertainty case. Therefore, it is imperative that uncertainty be taken into account during the optimisation of schedules for HEN maintenance problems.

\section{Acknowledgements}

This research is supported by the Ministry of Higher Education in the Sultanate of Oman and Petroleum Development Oman (PDO). This support is gratefully acknowledged.

\section{Appendix A}

There is a linear relation between the heat duty and the stream inlet and outlet temperatures. This is via the energy balances outlined in equations (A.1) and (A.2):

$$
Q=F_{\mathrm{c}} C_{\mathrm{c}}\left(T_{\mathrm{c}}^{\text {out }}-T_{\mathrm{c}}^{\mathrm{in}}\right)
$$




$$
Q=F_{\mathrm{h}} C_{\mathrm{h}}\left(T_{\mathrm{h}}^{\text {in }}-T_{\mathrm{h}}^{\text {out }}\right)
$$

where $F_{\mathrm{h}}$ and $F_{\mathrm{c}}$ are the mass flow-rates of the hot and cold streams, respectively and $C_{\mathrm{h}}$ and $C_{\mathrm{c}}$ are their specific heat capacities.

The cleaning scheduling problem is a multi-period decision-making OCP. A decision must be made regarding in which period(s) cleaning should occur and which unit(s) should be cleaned. Time is discretised into periods of equal length and each period is discretised further into a cleaning and operating sub-period. A control action represented by binary variable $y_{n, p}$ is defined to describe the cleaning status of each exchanger in each cleaning sub-period, where

$$
y_{n, p}=\left\{\begin{array}{cc}
0 & \text { if the } n \text {th heat exchanger is cleaned in period } p \\
1 & \text { otherwise }
\end{array} \quad \forall n, p\right.
$$

The control action is fixed to 1 when all units are online, i.e. within an operating sub-period. Over a specified operating horizon, $t_{\mathrm{F}}$, the objective is to minimise the overall operating and cleaning costs due to fouling. The objective is given by equation (A.4), which is generally common to all approaches.

$$
O b j=\int_{0}^{t_{\mathrm{F}}} \frac{C_{\mathrm{E}} Q_{\mathrm{F}}(t)}{\eta_{\mathrm{f}}} d t+\sum_{p=1}^{N P} \sum_{n=1}^{N E} C_{\mathrm{c}}\left(1-y_{n, p}\right)
$$

Here, $Q_{\mathrm{F}}(t)$ represents the extra furnace duty consumption due to fouling, which is based on the crude inlet temperature (CIT); $C_{\mathrm{E}}$ is the cost of fuel, $\eta_{\mathrm{f}}$ is the furnace efficiency; $N E$ is the total number of exchangers considered for cleaning; and $C_{\mathrm{c}}$ is the cost per cleaning action. By incorporating $y_{n, p}$, equations (7) and (8) become:

$$
\begin{gathered}
\dot{R}_{\mathrm{f}}=y_{n, p} a \quad \forall n, p \\
R_{\mathrm{f}}=R_{\mathrm{f}}^{\infty}\left(1-\exp \left(-t^{\prime} / \tau\right)\right)
\end{gathered}
$$




$$
\dot{t}^{\prime}=y_{n, p} \quad \forall n, p
$$

Initially, in the first period the fouling resistance is set to 0 for all exchangers, i.e. all units start from a clean condition. In subsequent periods, the fouling resistance is related to that at the end of the previous period. When cleaning takes place, this value is reinitialised via a junction condition.

The performance of the simple countercurrent heat exchangers considered is determined using the NTU-effectiveness approach. The temperatures of the cold and hot streams leaving an exchanger can be calculated by:

$$
\begin{gathered}
T_{\mathrm{c}}^{\text {out }}=T_{\mathrm{c}}^{\text {in }}+P\left(T_{\mathrm{c}}^{\text {in }}-T_{\mathrm{c}}^{\text {out }}\right) \\
T_{\mathrm{h}}^{\text {out }}=y_{n, p}\left[\frac{(1-P) T_{\mathrm{h}}^{\text {in }} \exp (-\alpha(1-P))+T_{\mathrm{c}}^{\text {in }}(1-\exp (-\alpha(1-P)))}{1-P \exp (-\alpha(1-P))}\right]+\left(1-y_{n, p}\right) T_{\mathrm{h}}^{\text {in }} \\
\forall n, p
\end{gathered}
$$

where the effectiveness term, $\alpha$ and the ratio of capacity flowrates, $P$, are (Smaïli et al., 2001):

$$
\begin{gathered}
\alpha=\frac{U A}{F_{\mathrm{h}} C_{\mathrm{h}}} \\
P=\frac{F_{\mathrm{h}} C_{\mathrm{h}}}{F_{\mathrm{c}} C_{\mathrm{c}}}
\end{gathered}
$$

Equations (A.7) and (A.8) are applicable to configurations with $P<1$. In a crude PHT, the cold (crude) stream usually has a significantly higher flow rate than the hot stream. 


\section{References}

Al Ismaili, R., Lee, M.W., Wilson, D.I., Vassiliadis, V.S., 2018. Heat exchanger network cleaning scheduling: From optimal control to mixed-integer decision making. Computers \& Chemical Engineering 111, 1-15. doi:10.1016/j.compchemeng.2017.12.004.

Alt, W., Schneider, C., Seydenschwanz, M., 2016. Regularization and implicit Euler discretization of linear-quadratic optimal control problems with bang-bang solutions. Applied Mathematics and Computation 287-288, 104-124. doi:10.1016/j.amc.2016.04.028.

Babuška, I., Silva, R.S., 2014. Dealing with uncertainties in engineering problems using only available data. Computer Methods in Applied Mechanics and Engineering 270, 57-75.

Beale, E..M..L.., 1955. On minimizing a convex function subject to linear inequalities. Journal of the Royal Statistical Society. Series B 17, 173-184.

Belghith, S.F., Lamnabhi-Lagarrigue, F., Rosset, M.M., 1986. Algebraic and geometric methods in nonlinear control theory. volume 29. Springer Netherlands, Dordrecht. doi:10.1007/978-94-009-4706-1.

Bellman, R., Glicksberg, I., Gross, O., 1956. On the bang-bang control problem. Quaterly of Applied Mathematics 14, 11-18.

Blakemore, N., Aris, R., 1962. Studies in optimization-V. The bang-bang control of a batch reactor. Chemical Engineering Science 17, 591-598.

Bryson, A.E., Ho, Y.C., 1975. Applied optimal control: Optimization, estimation and control. Hemisphere Publishing Corporation, New York-Washington-Philadelphia-London.

Dantzig, G.B., 1955. Linear Programming under Uncertainty. Management Science 1, 197206. doi:10.1287/mnsc.1.3-4.197.

Diwekar, U.M., 2003. Optimization under uncertainty in chemical engineering. Proceedings of the Indian National Science Academy 69 A, 267-284. 
El Amraoui, A., Mesghouni, K., 2014. Train scheduling networks under time duration uncertainty. volume 19. IFAC. doi:10.3182/20140824-6-ZA-1003.02200.

Galetakis, M., Roumpos, C., Alevizos, G., Vamvuka, D., 2012. Production scheduling of a lignite mine under quality and reserves uncertainty. Reliability Engineering and System Safety 107, 224-230. doi:10.1016/j.ress.2012.08.001.

Georgiadis, M.C., Papageorgiou, L.G., 2000. Optimal energy and cleaning management in heat exchanger networks under fouling. Chemical Engineering Research and Design 78, $168-179$.

Gonçalves, C.D.O., Queiroz, E.M., Pessoa, F.L.P., Liporace, F.S., Oliveira, S.G., Costa, A.L.H., 2014. Heuristic optimization of the cleaning schedule of crude preheat trains. Applied Thermal Engineering 73, 1-12. doi:10.1016/j.applthermaleng.2014.07.036.

Hu, Z., Hu, G., 2016. A two-stage stochastic programming model for lot-sizing and scheduling under uncertainty. International Journal of Production Economics 180, 198-207. doi:10.1016/j.ijpe.2016.07.027.

Ishiyama, E.M., Falkeman, E.S., Wilson, D.I., Pugh, S.J., 2017. Quantifying implications of deposit aging from crude refinery preheat train data, in: Heat Exchanger Fouling and Cleaning XII, Aranjuez. pp. 5-13.

Ishiyama, E.M., Paterson, W.R., Wilson, D.I., 2009. Platform for techno-economic analysis of fouling mitigation options in refinery preheat trains. Energy and Fuels 23, 1323-1337. doi:10.1021/ef8005614.

Ishiyama, E.M., Paterson, W.R., Wilson, D.I., 2011. Optimum cleaning cycles for heat transfer equipment undergoing fouling and ageing. Chemical Engineering Science 66, 604612. doi:10.1016/j.ces.2010.10.036. 
Kenan, N., Jebali, A., Diabat, A., 2017. An integrated flight scheduling and fleet assignment problem under uncertainty. Computers \& Operations Research, In Pressdoi:10.1016/j.cor.2017.08.014.

Lavaja, J.H., Bagajewicz, M.J., 2004a. Managing financial risk in the planning of heat exchanger cleaning, in: European Symposium on Computer-Aided Process Engineering14, Lisbon. pp. 235-240. doi:10.

Lavaja, J.H., Bagajewicz, M.J., 2004b. On a new MILP model for the planning of heatexchanger network cleaning. Industrial \& Engineering Chemistry Research 43, 3924-3938.

Lavaja, J.H., Bagajewicz, M.J., 2005. On a new MILP model for the planning of heatexchanger network cleaning. Part III: multiperiod cleaning under uncertainty with financial risk management. Industrial \& Engineering Chemistry Research 44, 8136-8146. doi:10.1021/ie0503186.

Lusa, A., Corominas, A., Muñoz, N., 2008. A multistage scenario optimisation procedure to plan annualised working hours under demand uncertainty. International Journal of Production Economics 113, 957-968. doi:10.1016/j.ijpe.2007.11.009.

Ma, R.S.T., Epstein, N., 1981. Optimum Cycles For Falling Rate Processes. The Canadian Journal of Chemical Engineering 59, 631-633. doi:10.1002/cjce.5450590512.

Mohler, R., 1973. Bilinear control processes: With applications to engineering, ecology and medicine. Academic Press, New York.

Müller-Steinhagen, H., 2000. Heat exchanger fouling: Mitigation and cleaning techniques. IChemE.

Müller-Steinhagen, H., Malayeri, M.R., Watkinson, A.P., 2005. Fouling of heat exchangersnew approaches to solve an old problem. Heat Transfer Engineering 26, 1-4. doi:10.1080/01457630590889906. 
Prasad, R., Karmeshu, Bharadwaj, K., 2002. Stochastic modeling of heat exchanger response to data uncertainties. Applied Mathematical Modelling 26, 715-726.

Pugh, S., Hewitt, G.F., Müller-Steinhagen, H., 2002. Heat exchanger fouling in the pre-heat train of a crude oil distillation unit - the development of a 'user guide', in: Proceedings of the 4th International Conference on Heat Exchanger Fouling, Fundamental Approaches \& Technical Solutions, Davos.

Sager, S., 2009. Reformulations and algorithms for the optimization of switching decisions in nonlinear optimal control. Journal of Process Control 19, 1238-1247. doi:10.1016/j.jprocont.2009.03.008.

Sel, Ç., Bilgen, B., Bloemhof-Ruwaard, J., 2017. Planning and scheduling of the make-andpack dairy production under lifetime uncertainty. Applied Mathematical Modelling 51, 129-144. doi:10.1016/j.apm.2017.06.002.

Sharqawy, M.H., Zubair, S.M., 2010. Heat exchangers design under variable overall heat transfer coefficient: Improved analytical and numerical approaches. Heat Transfer Engineering 31, 1051-1056. doi:10.1080/01457631003640313.

Sheikh, A.K., Zubair, S.M., Haq, M.U., Budair, M.O., 1996. Reliability-Based Maintenance Strategies for Heat Exchangers Subject to Fouling. Journal of Energy Resources Technology 118, 306. doi:10.1115/1.2793878.

Smaïli, F., Angadi, D.K., Hatch, C.M., Herbert, O., Vassiliadis, V.S., Wilson, D.I., 1999. Optimization of scheduling of cleaning in heat exchanger networks subject to fouling. Food and Bioproducts Processing 77, 159-164. doi:10.1205/096030899532312.

Smaïli, F., Vassiliadis, V.S., Wilson, D.I., 2001. Mitigation of fouling in refinery heat exchanger networks by optimal management of cleaning. Energy \& Fuels 15, 1038-1056. doi:10.1021/ef010052p. 
Smaïli, F., Vassiliadis, V.S., Wilson, D.I., 2002a. Long-term scheduling of cleaning of heat exchanger networks. Chemical Engineering Research and Design 80, 561-578. doi: $10.1205 / 026387602760312764$.

Smaïli, F., Vassiliadis, V.S., Wilson, D.I., 2002b. Optimization of cleaning schedules in heat exchanger networks subject to fouling. Chemical Engineering Communications 189, 1517-1549. doi:10.1080/00986440214999.

The MathWorks Inc., 2016. MATLAB and optimisation toolbox.

Vassiliadis, V.S., 1993. Computational solution of dynamic optimization problems with general differential-algebraic constraints. Ph.D. thesis. Imperial College London.

Wilson, D.I., Ishiyama, E.M., Polley, G.T., 2017. Twenty years of Ebert and Panchal—what next? Heat Transfer Engineering 38, 669-680. doi:10.1080/01457632.2016.1206407.

Yan, H., Zhu, Y., 2017. Bang-bang control model with optimistic value criterion for uncertain switched systems. Journal of Intelligent Manufacturing 28, 527-534. doi:10.1007/s10845014-0996-2. 